\title{
INFLUENCE OF GLUTATHIONE ADDITION TO AN EXTENDER ON THE COOLED- STORED STALLION SPERMATOZOA
}

\author{
ZEIDAN, A.E.B. ${ }^{1}$; A.A. EL-SHARABASSY ${ }^{2}$; M.Y. MEKKAWY ${ }^{2}$; G.M.A. SOLOUMA ${ }^{3}$; \\ A.M. AMER ${ }^{1}$; E.A.A. AHMADI ${ }^{1}$; R.M. ABOU-AIANA ${ }^{1}$ and I.A. EL-NADY ${ }^{2}$ \\ ${ }^{1}$ Animal Production Research Institute, Dokki, Giza, Egypt \\ ${ }^{2}$ Animal Production Department, Faculty of Agriculture, Cairo, Al-Azhar University, Egypt \\ ${ }^{3}$ Department of Animal Production, Faculty of Agriculture, Souhag, University, Egypt.
}

Received: 30 June 2019; Accepted: 30 July 2019

\begin{abstract}
Eighteen ejaculates were collected from three mature Arabian stallions (about 5 years of age) during breeding season (summer). Semen was collected, evaluated and extended with Tris-yolk-glucose, Milk-glucose and Nonfat Dried milk solids glucose extenders. The final extension rate was $1 \mathrm{ml}$ semen: $4 \mathrm{ml}$ extender. The extended semen was divided into 4 cervical tubes added with Glutathion (GSH) $0.0,0.2,0.4$ and $0.8 \mathrm{mM} / 100 \mathrm{ml}$ ، . The extended semen was kept at $5{ }^{\circ} \mathrm{C}$ for up to 48 hours. The percentages of sperm motility, dead spermatozoa, abnormal spermatozoa, and acrosome damage of spermatozoa were recorded. The results showed that, the percentage of motile stallion spermatozoa extended with tris-yolk-glucose or Non-fat Dried milk solids glucose extenders significantly $(\mathrm{P}<0.05)$ higher than Milk-glucose extender, while the percentages of dead spermatozoa, abnormal spermatozoa and acrosome damage of spermatozoa $(\%)$ significantly $(\mathrm{P}<0.05)$ lower than Milkglucose extender. Supplementation of GSH at level of $0.4 \mathrm{mM}$ to the extender increased significantly $(\mathrm{P}<0.05)$ the percentage of motile stallion spermatozoa, while decreased the percentages of dead spermatozoa, abnormal spermatozoa and acrosome damage of spermatozoa (\%) as compared with the control samples in the different extenders, during storage at $5^{\circ} \mathrm{C}$. The prolongation of storage time at $5^{\circ} \mathrm{C}$ decreased significantly $(\mathrm{P}<0.05)$ the percentages of sperm motility and increased significantly $(\mathrm{P}<0.05)$ the percentages of dead spermatozoa, abnormal spermatozoa and acrosome damage of spermatozoa (\%) in the different extenders or GSH concentrations and free-GSH medium (control). In conclusion, it can be recommended to use of Tris-yolkglucose extender or Non-fat Dried milk solids-glucose extender added with $0.4 \mathrm{mM}$ glutathione $/ 100 \mathrm{ml}$ for enhancement of the Arabian stallion spermatozoa, during storage at $5{ }^{\circ} \mathrm{C}$.
\end{abstract}

Key words: stallion, semen, extenders, glutathione.

\section{INTRODUCTION}

The horses were an important part of human life in prehistoric times. Horse domestication was a great breakthrough, bringing horsepower communications, transportation, farming and warfare. It has been estimated that only $24 \%$ of stallions produce ejaculates that are suitable for cryopreservation and fertility for frozen semen is approximately $40 \%$ compared to that of fresh. In stallions, motility characteristics are poorly correlated with fertility Jasko et al. (1992). The cryopreservation of gametes is expensive and requires sophisticated equipment. However, pregnancy rates remain low due to many variables Miller, (2008). As an arising technology, the implementation relies on the cost and feasibility, and may not be suitable to all breeding farms. Alternative procedures have been used with success to freeze semen immediately post-collection, but this requires the stallion and equipment be at the

Corresponding author: Dr. G.M.A. Solouma

E-mail address: gsolouma2@hotmail.com

Present address: Department of Animal Production, Faculty of Agriculture, Souhag, University, Egypt. same place. The collection and shipment of cooled semen to a specialized facility makes the cryopreservation process easier, without transporting the stallion to reproduction centers Crockett et al. (2001). However, manipulation of equine semen during these processes reduces sperm viability and fertility due to several factors including membrane lipid peroxidation, because of its high polyunsaturated fatty acids content, making spermatozoa highly susceptible to free radicals and reactive oxygen species (ROS; Cocchia et al., 2011).

ROS scavengers are present in seminal plasma, with the primary ROS scavengers described in equine semen being glutathione peroxidase, superoxide dismutase, and catalase. Bozkurt et al. (2007) found that the stallion spermatozoa diluted in milk-based extender had greater motility and longevity than those diluted in TRIS-based and glucose-lactose extenders, Oliveira et al. (2014) found that the addition of $2 \mathrm{mM}$ glutathione to the freezing extender increased the total motility, viability, and plasmatic membrane integrity of stallion spermatozoa de Oliveira et al. (2015) found also that the addition of glutathione at 
levels of 1.5 or $2.5 \mathrm{mM}$ were more efficient in protecting the sperm cells and yielded higher total motility after 36 hours of refrigeration. Glutathione is one of the antioxidants added to different semen specimens. It is a thiol tripeptide (yglutamyl cysteinyl glycine) with several biological functions found widely in the animal body, not only in somatic cells but also in gametes as well. This thiol has an important role in the ant oxidation process of endogenous and exogenous composts, as well as, in the maintenance of intracellular redox conditions. Glutathione is a natural reservoir of redox force, which can be quickly used by defend cells against oxidative stress Luberda (2005). It is synthesized from glutamate, cysteine, and glycine amino acids. Its reductive power is used to maintain thiol groups in intracellular proteins and other molecules. It acts as a cysteine physiological reservoir and is involved in the regulation of protein synthesis, cellular detoxification, and leukotriene synthesis. The protection by glutathione against oxidative damage is provided by its sulphydryl group (SH), which can be present in reduced glutathione $(\mathrm{GSH})$ and oxidized glutathione (GSSG) forms. The GSH's attack against ROS is favored by the interaction with enzymes, such as glutathione reductase and glutathione peroxidase Luberda, (2005). The thiol antioxidant system is represented mainly by glutathione, the primary antioxidant in equine semen, abundant in seminal plasma. The amount of GSH in equine seminal plasma is 10 times higher than that in swine Luberda, (2005). The best result was obtained with $0.4 \mathrm{mM}$ GSH level with egg yolk extender when stored up to $12 \mathrm{~h}$ at $25^{\circ} \mathrm{C}$ in ram Solouma (2013).

Therefore, the present study aimed to study the effect of glutathione concentrations $(0.0,0.2,0.4$ and 0.8 $\mathrm{mM} / 100 \mathrm{ml}$ ) added to different extenders (Tris-yolkglucose extender, Milk-glucose extender and Non-fat Dried milk solids glucose extender) on semen quality of the Arabian stallions, during storage at $5^{\circ} \mathrm{C}$ for up to 48 hours.

\section{MATERIALS AND METHODS}

The present study was conducted in the Animal Production Department, Faculty of Agriculture, AlAzhar University, Cairo, and Animal Production Research Institute, Agricultural Research Center, Egypt. The experimental work was carried out in Arabian stallions reared in E1-Zahra Governmental Stud, Ain-Shams Town, nearby Cairo, Egypt, during the period from March, 2015 till February, 2016. In the present study, the ejaculates of semen were collected from three healthy, intact and sexually matured (5 years of age) of Arabian stallions, during breeding season.

\section{Semen collection:}

Semen was collected once per week from each stallion by means of an Artificial Vagina (AV) using Missouri Model, while stallions mounted at mare in estrous. The AV was pre-warmed to $45-50^{\circ} \mathrm{C}$, fitted with a nylon filter coupled to a collection cup to obtain the gel-free fraction. The collected ejaculate was immediately transported to the laboratory for evaluation and processing.

\section{Semen extension and glutathione addition:}

Semen was evaluated immediately after collection then extended with the different extenders (Tris-yolkglucose extender, Milk-glucose extender and Non-fat Dried milk solids-glucose extender). The extended semen was divided into 4 cervical tubes added with Glutathion (GSH) at levels which are Control and three treatments Respectively: $0.0,0.2,0.4$ and 0.8 $\mathrm{mM} / 100 \mathrm{ml}$. The compositions of these extenders are shown in Table 1 according to Mckinnon and Voss (1993). Semen extension was carried out by adding the appropriate volume of the extender slowly to the semen. Extended semen (in tube) was kept below the level of water in water bath at $37^{\circ} \mathrm{C}$ for all times to avoid fluctuations in the temperature of the extended semen. The final extension rate was 1semen: 4 extender.

Table 1: Composition of buffered yolk extenders

\begin{tabular}{ccc}
\hline Extender & Ingredients & Quantity $\mathbf{( g m / 1 0 0 m l ~ d i s t i l l e d ~ w a t e r ) ~}$ \\
\hline \multirow{3}{*}{ Tris-yolk-glucose extender } & ${ }^{*}$ Tris aminomethan & 2.40 \\
\cline { 2 - 3 } & Glucose monohydrate & 0.45 \\
\cline { 2 - 3 } & Citric acid anhydrous & 1.25 \\
\cline { 2 - 3 } Milk-glucose extender & Egg yolk (ml) & 22.00 \\
\cline { 2 - 3 } & Dry skim milk & 2.40 \\
\cline { 2 - 3 } & Glucose monohydrate & 4.90 \\
\hline \multirow{2}{*}{$\begin{array}{c}\text { Non-fat Dried milk solids } \\
\text { glucose extender }\end{array}$} & Glucose monohydrate & 2.00 \\
\cline { 2 - 3 } & Sodium bicarbonate (7.5\% solution) & 2.40 \\
\cline { 2 - 3 } & Sodiun & 4.90 \\
\hline
\end{tabular}

$500 \mathrm{IU} / \mathrm{ml}$ penicillin $+500 \mu \mathrm{g} / \mathrm{ml}$ streptomycin sulphate were added to each extender.

*Tris(hydroxymethyl) aminomethan, Aldrich Chemical Co.Ltd., Gillingham, Dorest England.

* Sanalac (instant Non-fat dry milk). 


\section{Chilling of semen at $5^{\circ} \mathrm{C}$ :}

The test tubes containing extended semen were placed in a $500 \mathrm{ml}$ beaker containing water at $30^{\circ} \mathrm{C}$ with a thermometer in order to facilitate periodic checking of the water temperature during the cooling period. Another test tubes containing extended semen only (control tube) were placed in the same beaker to maintain the temperature similar to that of the semen (all the test tubes were covered with a dark plastic sheath). The beaker was placed in a refrigerator and gradually cooled till their temperature reached $5^{\circ} \mathrm{C}$ throughout 1.5-2.0 hours. The cooled semen with the different extenders was kept at $5^{\circ} \mathrm{C}$ for up to 0,24 and 48 hours.

After each storage time (0, 24 and 48 hours), the percentages of sperm motility, dead spermatozoa, abnormal spermatozoa and acrosome damage of spermatozoa with the different extenders and control were determined.

\section{Percentage of sperm motility:}

Sperm motility (\%) was estimated by adding one drop of gel-free semen on the dry, clean and pre-warmed $\left(37^{\circ} \mathrm{C}\right)$ glass slide. The drop of semen was covered by a warmed cover slip and immediately examined using high power magnification $(400 \mathrm{x})$ sperm motility was estimated by observing the approximate percentage of spermatozoa moving forward motion across the field of vision with a normal vigorous swimming motion according to Salisbury et al. (1978).

\section{Percentage of dead spermatozoa:}

The eosin/nigrosine staining procedure was carried out according to Hackett and Macpherson (1965). The percentage of dead spermatozoa was calculated from 100 spermatozoa which were counted in each slide in different microscope fields using a hand counter.

\section{Percentage of abnormal spermatozoa:}

The morphological of abnormal spermatozoa (\%) were determined by counting 100 sperm in the same smear prepared for live/dead spermatozoa ratio under oil immersion using X1000 objective of a light microscope according to Salisbary et al. (1978).

\section{Percentage of acrosome damage:}

Assessment of the percentage of acrosome damage of spermatozoa was done according to Watson (1975). A drop of diluted semen was smeared on a pre-warmed slide and allowed to dry in the current air. The smears were fixed by immersion in buffered formalin saline $(9 \mathrm{ml}$ sodium chloride $+100 \mathrm{ml}$ formalin $+900 \mathrm{ml}$ distilled water) for 15 minutes and then washed in running tap water for 15 to 20 minutes. The slides were dried and then immersed in buffered Giemsa stain solution for 90 minutes and after that they were rinsed briefly in distilled water and dried. A stock of Giemsa stain solution was prepared from solid Giemsa stain (Northampton, U.K. P460 D) as follows: Giemsa stain (3.8 g) was ground with absolute methanol (AR grade, $375 \mathrm{ml}$ ). Glycerol (AR grade, $125 \mathrm{ml}$ ) was added and stored at $37^{\circ} \mathrm{C}$ for one week. The percentage of acrosome damage was calculated for 100 spermatozoa observed at random on each slide using oil immersion lens (x1000).

\section{Sperm-cell concentration $\left(\times 10^{6} / \mathrm{ml}\right)$ :}

The spermatozoa were counted using Haemeocytomter according to Jasko et al. (1992).

\section{Statistical analysis:}

Data were statistically analyzed using the General Linear Model (SAS, 2008) one way analysis of variance. All statements of significant difference are based on the 0.05 or 0.01 probability levels. Duncan's New Multiple Range Test (Duncan, 1955) was used to detect differences among means. The percentage values were transformed to arcsine values before being statistically analyzed.

The following model was used:

Yij $=\mu+\mathrm{Ti}+$ Eij

Where,

Yij $=$ Experimental observation. $\mu=$ The overall mean.

$\mathrm{Ti}=$ The effect of Glutathione levels, $\mathrm{i}=0.0,0.2,0.4$ and $0.8 \mathrm{mM} / 100 \mathrm{ml}$

Eij $=$ The errors related to individual observation.

\section{RESULTS}

\section{Percentage of motile stallion spermatozoa:}

Data presented in Table 2 revealed that, the percentage of motile stallion spermatozoa in trisyolk-glucose extender and Non-fat Dried milk solidsglucose extender were significantly $(\mathrm{P}<0.05)$ higher than Milk-glucose extender. Supplementation of the extended stallion semen with glutathione up to 0.8 $\mathrm{mM} / 100 \mathrm{ml}$ increased significantly $(\mathrm{P}<0.05)$ the percentage of sperm motility, during storage at $5^{\circ} \mathrm{C}$ for up to 48 hours in the different extenders. Among GSH supplementation levels, the highest $(\mathrm{P}<0.05)$ percentage of sperm motility was recorded with the semen containing $0.4 \mathrm{mM}$ of GSH in the different extenders. While, the lowest $(\mathrm{P}<0.05)$ percentage of sperm motility was recorded with free-GSH medium (control) in the different extenders. 
Table 2: Mean percentage of motile Arabian stallion spermatozoa with different extenders and glutathione levels, during storage at $5^{\circ} \mathrm{C}$ for up to 48 hours.

\begin{tabular}{|c|c|c|c|c|c|c|c|c|c|c|c|c|c|c|c|}
\hline \multirow{3}{*}{$\begin{array}{c}\text { Storage } \\
\text { times } \\
\text { (hours) }\end{array}$} & \multicolumn{5}{|c|}{ Tris-yolk-glucoses } & \multicolumn{5}{|c|}{ Milk-glucose } & \multicolumn{5}{|c|}{ Non-fat Dried milk solids-glucose } \\
\hline & Gluta & hione lev & $\operatorname{vel}(\mathrm{m})$ & $/ 100 \mathrm{ml})$ & Means & Glutatl & hione lev & el $(\mathbf{m M})$ & $/ 100 \mathrm{ml})$ & Means & Glutat & hione le & $\mathbf{l}(\mathbf{m l})$ & $/ 100 \mathrm{ml})$ & Means \\
\hline & 0 & 0.2 & 0.4 & 0.8 & & 0 & 0.2 & 0.4 & 0.8 & & 0 & 0.2 & 0.4 & 0.8 & \\
\hline 0 & $\begin{array}{r}79.23 \\
\pm 1.34\end{array}$ & $\begin{array}{l}87.32 \\
\pm 1.89\end{array}$ & $\begin{array}{l}91.42 \\
\pm 2.01\end{array}$ & $\begin{array}{l}89.16 \\
\pm 1.32\end{array}$ & $\begin{array}{c}86.78^{\mathrm{A}} \\
\pm 1.28\end{array}$ & $\begin{array}{l}68.68 \\
\pm 1.82\end{array}$ & $\begin{array}{l}75.26 \\
\pm 1.59\end{array}$ & $\begin{array}{l}80.71 \\
\pm 1.72\end{array}$ & $\begin{array}{r}76.24 \\
\pm 1.65\end{array}$ & $\begin{array}{c}75.22^{\mathrm{A}} \\
\pm 1.04\end{array}$ & $\begin{array}{l}76.21 \\
\pm 1.58\end{array}$ & $\begin{array}{l}85.16 \\
\pm 1.75\end{array}$ & $\begin{array}{l}89.81 \\
\pm 1.42\end{array}$ & $\begin{array}{l}87.15 \\
\pm 1.34\end{array}$ & $\begin{array}{c}84.58^{A} \\
\pm 1.26\end{array}$ \\
\hline 24 & $\begin{array}{l}71.82 \\
\pm 1.78\end{array}$ & $\begin{array}{l}80.41 \\
\pm 1.82\end{array}$ & $\begin{array}{l}84.79 \\
\pm 1.92\end{array}$ & $\begin{array}{l}82.65 \\
\pm 2.11\end{array}$ & $\begin{array}{c}79.91^{\mathrm{B}} \\
\pm 1.77^{2}\end{array}$ & $\begin{array}{l}59.19 \\
\pm 1.78\end{array}$ & $\begin{array}{l}67.15 \\
\pm 1.81\end{array}$ & $\begin{array}{l}71.24 \\
\pm 1.73\end{array}$ & $\begin{array}{r}68.32 \\
\pm 1.86\end{array}$ & $\begin{array}{c}66.47^{B} \\
\pm 1.12\end{array}$ & $\begin{array}{l}68.13 \\
\pm 1.34\end{array}$ & $\begin{array}{l}75.24 \\
\pm 1.88\end{array}$ & $\begin{array}{l}83.61 \\
\pm 1.74\end{array}$ & $\begin{array}{l}80.29 \\
\pm 1.58\end{array}$ & $\begin{array}{c}76.81^{B} \\
\pm 1.18\end{array}$ \\
\hline 48 & $\begin{array}{l}56.13 \\
\pm 1.48\end{array}$ & $\begin{array}{l}62.19 \\
\pm 1.32\end{array}$ & $\begin{array}{l}68.58 \\
\pm 1.71 \\
\end{array}$ & $\begin{array}{l}64.28 \\
\pm 1.28\end{array}$ & $\begin{array}{c}62.79^{\mathrm{C}} \\
\pm 1.75\end{array}$ & $\begin{array}{l}43.28 \\
\pm 1.16\end{array}$ & $\begin{array}{l}50.33 \\
\pm 1.80\end{array}$ & $\begin{array}{l}54.26 \\
\pm 1.33\end{array}$ & $\begin{array}{l}52.37 \\
\pm 1.42 \\
\end{array}$ & $\begin{array}{c}50.06^{\mathrm{C}} \\
\pm 1.48\end{array}$ & $\begin{array}{l}57.85 \\
\pm 1.73\end{array}$ & $\begin{array}{l}64.70 \\
\pm 1.62 \\
\end{array}$ & $\begin{array}{l}67.12 \\
\pm 1.77\end{array}$ & $\begin{array}{l}65.42 \\
\pm 1.82 \\
\end{array}$ & $\begin{array}{c}63.77^{\mathrm{C}} \\
\pm 1.30\end{array}$ \\
\hline Means & $\begin{array}{c}69.06^{d} \\
\pm 1.11\end{array}$ & $\begin{array}{c}76.64^{b c} \\
\pm 1.16\end{array}$ & $\begin{array}{c}81.59^{a} \\
\pm 1.98\end{array}$ & $\begin{array}{c}78.69^{b} \\
\pm 1.04\end{array}$ & $76.49^{\mathrm{a}}$ & $\begin{array}{c}57.05^{b} \\
\pm 2.16\end{array}$ & $\begin{array}{c}64.24^{b c} \\
\pm 1.82\end{array}$ & $\begin{array}{c}68.73^{a} \\
\pm 1.94\end{array}$ & $\begin{array}{c}65.64 \\
\pm 1.98^{b}\end{array}$ & $63.91^{b}$ & $\begin{array}{c}67.39^{d} \\
\pm 1.08\end{array}$ & $\begin{array}{c}75.03^{b c} \\
\pm 1.89\end{array}$ & $\begin{array}{c}80.18^{a} \\
\pm 1.92\end{array}$ & $\begin{array}{c}77.62^{b} \\
\pm 1.03\end{array}$ & $\mathbf{7 5 . 0 5} \mathbf{5}^{\mathrm{a}}$ \\
\hline
\end{tabular}

A-C: Means with the different superscripts in the same column, differ significantly $(\mathrm{P}<0.05)$.

a-d: Means with the different superscripts in the same row, differ significantly $(\mathrm{P}<0.05)$.

The advancement of storage time at $5^{\circ} \mathrm{C}$ for up to 48 hours decreased significantly $(\mathrm{P}<0.05)$ the sperm motility in the different extenders or GSH levels

\section{Percentage of dead spermatozoa:}

Data presented in Table 3 showed significantly $(\mathrm{P}<0.05)$ lower in the percentage of dead spermatozoa in Tris-yolk-glucose extender and Non-fat Dried milk solids-glucose extender than Milk-glucose extender. supplementation of the extended stallion spermatozoa with GSH with $0.8 \mathrm{mM} / 100 \mathrm{ml}$ decreased significantly $(\mathrm{P}<0.05)$ the percentage of dead spermatozoa, during storage at $5^{\circ} \mathrm{C}$ for up to 48 hours in the different extenders. The highest $(\mathrm{P}<0.05)$ percentage of dead spermatozoa was recorded with free-semen GSH (control) and the lowest $(\mathrm{P}<0.05)$ value was recorded with the extended semen added with $0.4 \mathrm{mM} \mathrm{GSH}$ in the different extenders

Table 3: Mean percentage of dead Arabian stallions spermatozoa with different extenders and glutathione levels, during storage at $5^{\circ} \mathrm{C}$ for up to 48 hours

\begin{tabular}{|c|c|c|c|c|c|c|c|c|c|c|c|c|c|c|c|}
\hline \multirow{3}{*}{$\begin{array}{l}\text { Storage } \\
\text { times } \\
\text { (hours) }\end{array}$} & \multicolumn{5}{|c|}{ Tris-yolk-glucose } & \multicolumn{5}{|c|}{ Milk- glucose } & \multicolumn{5}{|c|}{ Non-fat Dried milk solids-glucose } \\
\hline & \multicolumn{4}{|c|}{$\begin{array}{c}\text { Glutathione level } \\
(\mathrm{mM} / 100 \mathrm{ml})\end{array}$} & \multirow[t]{2}{*}{ Means } & \multicolumn{2}{|c|}{ Glutathione lev } & el $(\mathrm{mM}$ & 100ml) & \multirow[t]{2}{*}{ Means } & \multicolumn{4}{|c|}{ Glutathione level (mM/100ml) } & \multirow[t]{2}{*}{ Means } \\
\hline & 0 & 0.2 & 0.4 & 0.8 & & 0 & 0.2 & 0.4 & 0.8 & & 0 & 0.2 & 0.4 & 0.8 & \\
\hline 0 & $\begin{array}{l}19.80 \\
\pm 0.87\end{array}$ & $\begin{array}{l}14.48 \\
\pm 0.78\end{array}$ & $\begin{array}{c}6.53 \\
\pm 0.91\end{array}$ & $\begin{array}{c}9.72 \\
\pm 0.42\end{array}$ & $\begin{array}{l}12.63^{\mathrm{C}} \\
\pm 0.10\end{array}$ & $\begin{array}{l}28.16 \\
\pm 0.73\end{array}$ & $\begin{array}{l}20.11 \\
\pm 0.34\end{array}$ & $\begin{array}{l}14.26 \\
\pm 0.56\end{array}$ & $\begin{array}{l}15.30 \\
\pm 041\end{array}$ & $\begin{array}{l}19.45^{\mathrm{C}} \\
\pm 0.93\end{array}$ & $\begin{array}{l}21.43 \\
\pm 0.84\end{array}$ & $\begin{array}{l}15.34 \\
\pm 0.73\end{array}$ & $\begin{array}{c}7.11 \\
\pm 0.64\end{array}$ & $\begin{array}{c}9.81 \\
\pm 0.43\end{array}$ & $\begin{array}{l}13.42^{\mathrm{C}} \\
\pm 0.42\end{array}$ \\
\hline 24 & $\begin{array}{l}28.71 \\
\pm 0.79 \\
\end{array}$ & $\begin{array}{l}17.52 \\
\pm 0.91 \\
\end{array}$ & $\begin{array}{c}8.42 \\
\pm 0.84 \\
\end{array}$ & $\begin{array}{r}12.35 \\
\pm 0.43 \\
\end{array}$ & $\begin{array}{c}16.75^{\mathrm{B}} \\
\pm 0.14\end{array}$ & $\begin{array}{l}36.12 \\
\pm 0.52 \\
\end{array}$ & $\begin{array}{l}25.95 \\
\pm 0.74 \\
\end{array}$ & $\begin{array}{l}20.16 \\
\pm 0.28 \\
\end{array}$ & $\begin{array}{l}23.14 \\
\pm 0.34 \\
\end{array}$ & $\begin{array}{l}26.34^{B} \\
\pm 1.03 \\
\end{array}$ & $\begin{array}{l}29.23 \\
\pm 0.74 \\
\end{array}$ & & $\begin{array}{l}11.35 \\
\pm 0.43 \\
\end{array}$ & $\begin{array}{l}13.45 \\
\pm 0.28 \\
\end{array}$ & $\begin{array}{c}18.48^{\mathrm{B}} \\
\pm 0.62 \\
\end{array}$ \\
\hline 48 & $\begin{array}{l}35.05 \\
\pm 0.94\end{array}$ & $\begin{array}{l}22.73 \\
\pm 0.87\end{array}$ & $\begin{array}{l}12.42 \\
\pm 0.62\end{array}$ & $\begin{array}{l}18.53 \\
\pm 0.48\end{array}$ & $\begin{array}{l}22.18^{A} \\
\pm 0.17\end{array}$ & $\begin{array}{l}48.92 \\
\pm 1.16\end{array}$ & $\begin{array}{l}32.53 \\
\pm 0.94\end{array}$ & $\begin{array}{c}26.83 \pm \\
1.10\end{array}$ & $\begin{array}{l}28.46 \\
\pm 1.12\end{array}$ & $\begin{array}{l}34.18^{A} \\
\pm 1.02\end{array}$ & $\begin{array}{l}38.11 \\
\pm 0.82\end{array}$ & $\begin{array}{l}25.92 \\
\pm 0.78\end{array}$ & $\begin{array}{r}14.25 \\
\pm 0.51\end{array}$ & $\begin{array}{l}21.42 \\
\pm 0.32\end{array}$ & $\begin{array}{l}24.92^{A} \\
\pm 0.41\end{array}$ \\
\hline Means & $\begin{array}{l}27.85^{\mathrm{a}} \\
\pm 0.98\end{array}$ & $\begin{array}{l}18.24^{6} \\
\pm 0.65\end{array}$ & $\begin{array}{l}9.12^{\mathrm{d}} \\
\pm 0.72\end{array}$ & $\begin{array}{l}13.53^{\mathrm{c}} \\
\pm 0.81\end{array}$ & $7.18^{\mathrm{b}}$ & $\begin{array}{l}37.73^{\mathrm{a}} \\
\pm 1.11\end{array}$ & $\begin{array}{l}26.19^{b} \\
\pm 1.32\end{array}$ & $\begin{array}{l}20.41^{\mathrm{d}} \\
\pm 0.94\end{array}$ & $\begin{array}{l}22.30^{c} \\
\pm 0.87\end{array}$ & $5^{\mathrm{a}}$ & $\begin{array}{l}29.59^{\mathrm{a}} \\
\pm 0.42\end{array}$ & $\begin{array}{l}20.39^{b} \\
\pm 0.18\end{array}$ & $\begin{array}{l}10.90^{\mathrm{d}} \\
\pm 0.27\end{array}$ & $\begin{array}{l}14.89^{c} \\
\pm 0.42\end{array}$ & $18.94^{b}$ \\
\hline
\end{tabular}

A-C: Means with the different superscripts in the same column, differ significantly $(\mathrm{P}<0.05)$.

a-d: Means with the different superscripts in the same row, differ significantly $(\mathrm{P}<0.05)$.

Regarding the effect of storage time, the advancement of storage time at $5^{\circ} \mathrm{C}$ for up to 48 hours increased significantly $(\mathrm{P}<0.05)$ the percentage of dead stallion spermatozoa in the different extenders or GSH levels. The lowest $(\mathrm{P}<0.05)$ value of dead spermatozoa was recorded at zero time, while the highest $(\mathrm{P}<0.05)$ value was recorded at 48 hours with the different extenders

\section{Percentage of abnormal spermatozoa:}

Data presented in Table 4 revealed that, the percentage of abnormal stallion spermatozoa significantly $(\mathrm{P}<0.05)$ lower with Tris-yolk-glucose extender and Non-fat dried milk solid glucose extenders than Milk-glucose extender. Supplementation of the extended stallion semen with glutathione (GSH) up to $0.8 \mathrm{mM} / 100 \mathrm{ml}$ caused decreased significantly $(\mathrm{P}<0.05)$ the percentage of abnormal spermatozoa during storage at $5^{\circ} \mathrm{C}$ for up to 48 hours in the different extenders. The lowest $(\mathrm{P}<0.05)$ value of abnormal spermatozoa was recorded in the extended semen with $0.4 \mathrm{mM} \mathrm{GSH}$ and the highest $(\mathrm{P}<0.05)$ value was observed with semen free- GSH (control). 
Table 4: Mean percentage of abnormal spermatozoa of the Arabian stallions with different extenders and glutathione levels, during storage at $5^{\circ} \mathrm{C}$ for up to 48 hours

\begin{tabular}{|c|c|c|c|c|c|c|c|c|c|c|c|c|c|c|c|}
\hline \multirow{3}{*}{$\begin{array}{c}\text { Storage } \\
\text { times } \\
\text { (hours) }\end{array}$} & \multicolumn{5}{|c|}{ Tris-yolk-glucose } & \multicolumn{5}{|c|}{ Milk- glucose } & \multicolumn{5}{|c|}{ Non-fat Dried milk solids-glucose } \\
\hline & \multicolumn{4}{|c|}{$\begin{array}{c}\text { Glutathione level } \\
(\mathrm{mM} / \mathbf{1 0 0} \mathrm{ml})\end{array}$} & \multirow[t]{2}{*}{ Means } & \multicolumn{4}{|c|}{$\begin{array}{c}\begin{array}{c}\text { Glutathione level } \\
(\mathrm{mM} / 100 \mathrm{ml})\end{array} \\
\end{array}$} & \multirow[t]{2}{*}{ Means } & \multicolumn{4}{|c|}{$\begin{array}{c}\text { Glutathione level } \\
(\mathrm{mM} / 100 \mathrm{ml})\end{array}$} & \multirow[t]{2}{*}{ Means } \\
\hline & 0 & 0. & 0.4 & 0.8 & & 0 & 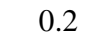 & 0.4 & 0.8 & &  & 0.2 & 0.4 & 0.8 & \\
\hline 0 & $\begin{array}{l}13.73 \\
\pm 0.93\end{array}$ & $\begin{array}{c}8.34 \\
\pm 0.41\end{array}$ & $\begin{array}{c}3.82 \\
\pm 0.27\end{array}$ & $\begin{array}{c}5.68 \\
\pm 0.34\end{array}$ & & $\begin{array}{l}23.42 \\
\pm 0.28\end{array}$ & $\begin{array}{l}15.25 \\
\pm 0.13\end{array}$ & $\begin{array}{c}9.14 \\
\pm 0.14\end{array}$ & $\begin{array}{l}10.44 \\
\pm 0.16\end{array}$ & & $\begin{array}{l}16.82 \\
\pm 0.14\end{array}$ & $\begin{array}{l}10.16 \\
\pm 0.11\end{array}$ & $\begin{array}{c}7.22 \\
\pm 0.13\end{array}$ & $\begin{array}{c}8.92 \\
\pm 0.16\end{array}$ & \\
\hline 24 & $\begin{array}{l}19.23 \\
\pm 0.84 \\
\end{array}$ & $\begin{array}{l}14.30 \\
\pm 0.72 \\
\end{array}$ & $\begin{array}{c}8.42 \\
\pm 0.58 \\
\end{array}$ & $\begin{array}{l}11.50 \\
\pm 0.62 \\
\end{array}$ & $\begin{array}{r}13.36^{\mathrm{B}} \\
\pm 0.56 \\
\end{array}$ & $\begin{array}{l}30.16 \\
\pm 0.64 \\
\end{array}$ & $\begin{array}{l}20.91 \\
\pm 0.71 \\
\end{array}$ & $\begin{array}{l}13.34 \\
\pm 0.65 \\
\end{array}$ & $\begin{array}{l}15.17 \\
\pm 0.41 \\
\end{array}$ & $\begin{array}{l}19.89^{\mathrm{B}} \\
\pm 0.22 \\
\end{array}$ & $\begin{array}{l}22.18 \\
\pm 0.24 \\
\end{array}$ & $\begin{array}{l}15.32 \\
\pm 0.14 \\
\end{array}$ & $\begin{array}{r}9.27 \\
\pm 0.16 \\
\end{array}$ & $\begin{array}{l}10.14 \\
\pm 0.18 \\
\end{array}$ & $\begin{array}{r}14.22^{\mathrm{B}} \\
\pm 0.19 \\
\end{array}$ \\
\hline 48 & $\begin{array}{l}26.72 \\
\pm 0.56\end{array}$ & $\begin{array}{l}21.43 \\
\pm 0.65\end{array}$ & $\begin{array}{r}12.24 \\
\pm 0.42\end{array}$ & $\begin{array}{l}16.22 \\
\pm 0.32\end{array}$ & $\begin{array}{r}19.15^{\mathrm{A}} \\
\pm 0.34\end{array}$ & $\begin{array}{l}37.25 \\
\pm 0.41\end{array}$ & $\begin{array}{l}26.24 \\
\pm 0.23\end{array}$ & $\begin{array}{l}18.27 \\
\pm 0.11\end{array}$ & $\begin{array}{l}22.40 \\
\pm 0.19\end{array}$ & $\begin{array}{l}26.04^{\mathrm{A}} \\
\pm 0.22\end{array}$ & $\begin{array}{l}28.24 \\
\pm 0.32\end{array}$ & $\begin{array}{l}22.31 \\
\pm 0.25\end{array}$ & $\begin{array}{l}13.26 \\
\pm 0.11\end{array}$ & $\begin{array}{l}17.52 \\
\pm 0.11\end{array}$ & $\begin{array}{c}20.33^{\mathrm{A}} \\
\pm 0.23\end{array}$ \\
\hline Means & $\begin{array}{l}19.89^{\mathrm{a}} \\
\pm 0.82\end{array}$ & $\begin{array}{l}14.69^{b} \\
\pm 0.61\end{array}$ & $\begin{array}{l}8.16^{b} \\
\pm 0.23\end{array}$ & $\begin{array}{l}11.13^{\mathrm{c}} \\
\pm 0.18\end{array}$ & $13.46^{b}$ & $\begin{array}{l}30.27^{\mathrm{a}} \\
\pm 0.87\end{array}$ & $\begin{array}{r}20.80^{b} \\
\pm 0.16\end{array}$ & $\begin{array}{r}13.58^{d} \\
\pm 0.19\end{array}$ & $\begin{array}{l}16.00^{c} \\
\pm 0.21\end{array}$ & $20.16^{\mathrm{a}}$ & $\begin{array}{l}22.41^{\mathrm{a}} \\
\pm 0.41\end{array}$ & $\begin{array}{l}15.93^{b} \\
\pm 0.16\end{array}$ & $\begin{array}{l}9.91^{\mathrm{d}} \\
\pm 0.14\end{array}$ & $\begin{array}{l}12.19^{c} \\
\pm 0.18\end{array}$ & $15.11^{\mathrm{b}}$ \\
\hline
\end{tabular}

A-C: Means with the different superscripts in the same column, differ significantly $(\mathrm{P}<0.05)$.

a-d: Means with the different superscripts in the same row, differ significantly $(\mathrm{P}<0.05)$.

Regarding the effect of storage time, the advancement of storage time at $5^{\circ} \mathrm{C}$ for up to 48 hours significantly increased $(\mathrm{P}<0.05)$ the percentage of abnormal stallion spermatozoa in different extenders and GSH levels $(0.2,0.4$ and $0.8 \mathrm{mM})$. The lowest $(\mathrm{P}<0.05)$ value of abnormal spermatozoa was recorded at zero time, while the highest $(\mathrm{P}<0.05)$ value was recorded at 48 hours with different extender.

4. Percentage of acrosome damage of spermatozoa:

Table 5: Mean percentage of acrosome damage of the Arabian stallions spermatozoa with the different extenders and glutathione levels, during storage at $5^{\circ} \mathrm{C}$ for up to 48 hours.

\begin{tabular}{|c|c|c|c|c|c|c|c|c|c|c|c|c|c|c|c|}
\hline \multirow{3}{*}{$\begin{array}{c}\text { Storage } \\
\text { times } \\
\text { (hours) }\end{array}$} & \multicolumn{5}{|c|}{ Tris-yolk-glucose } & \multicolumn{5}{|c|}{ Milk- glucose } & \multicolumn{5}{|c|}{ Non-fat Dried milk solids-glucose } \\
\hline & \multicolumn{4}{|c|}{$\begin{array}{l}\text { Glutathione level } \\
(\mathrm{mM} / \mathbf{1 0 0 m \mathrm { ml } )}\end{array}$} & \multirow[t]{2}{*}{ Means } & \multicolumn{4}{|c|}{ Glutathione level $(\mathrm{mM} / \mathbf{1 0 0 m l})$} & Means & \multicolumn{4}{|c|}{$\begin{array}{c}\text { Glutathione level } \\
(\mathrm{mM} / \mathbf{1 0 0 m l})\end{array}$} & \multirow[t]{2}{*}{ Means } \\
\hline & 0 & 0.2 & 0.4 & 0.8 & & 0 & 0.2 & 0.4 & 0.8 & & 0 & 0.2 & 0.4 & 0.8 & \\
\hline 0 & $\begin{array}{c}9.48 \\
\pm 0.83\end{array}$ & $\begin{array}{c}7.25 \\
\pm 0.76\end{array}$ & $\begin{array}{c}1.12 \\
\pm 0.45\end{array}$ & $\begin{array}{c}2.71 \\
\pm 0.53\end{array}$ & $\begin{array}{l}5.14^{\mathrm{C}} \\
\pm 0.38\end{array}$ & $\begin{array}{l}12.53 \\
\pm 0.16\end{array}$ & $\begin{array}{c}9.41 \\
\pm 0.38\end{array}$ & $\begin{array}{c}3.68 \\
\pm 0.19\end{array}$ & $\begin{array}{c}6.18 \\
\pm 0.11\end{array}$ & $\begin{array}{l}7.95^{\mathrm{C}} \\
\pm 0.18\end{array}$ & $\begin{array}{c}8.92 \\
\pm 0.12\end{array}$ & $\begin{array}{c}5.12 \\
\pm 0.10\end{array}$ & $\begin{array}{c}1.94 \\
\pm 0.11\end{array}$ & $\begin{array}{c}3.15 \\
\pm 0.16\end{array}$ & $\begin{array}{c}4.78^{C} \pm \\
0.17\end{array}$ \\
\hline 24 & $\begin{array}{l}15.34 \\
\pm 0.46 \\
\end{array}$ & $\begin{array}{l}12.16 \\
\pm 0.81 \\
\end{array}$ & $\begin{array}{r}2.19 \\
\pm 0.11 \\
\end{array}$ & $\begin{array}{r}7.87 \\
\pm 0.63 \\
\end{array}$ & $\begin{array}{c}9.39^{\mathrm{B}} \\
\pm 0.54 \\
\end{array}$ & $\begin{array}{l}19.46 \\
\pm 0.43 \\
\end{array}$ & $\begin{array}{r}15.27 \\
\pm 0.52 \\
\end{array}$ & $\begin{array}{c}6.72 \\
\pm 0.71 \\
\end{array}$ & $\begin{array}{r}11.51 \\
\pm 0.13 \\
\end{array}$ & $\begin{array}{c}13.24^{B} \\
\pm 0.26 \\
\end{array}$ & $\begin{array}{l}14.89 \\
\pm 0.13 \\
\end{array}$ & $\begin{array}{l}10.42 \\
\pm 0.19 \\
\end{array}$ & $\begin{array}{c}3.78 \\
\pm 0.16 \\
\end{array}$ & $\begin{array}{c}6.28 \\
\pm 0.12 \\
\end{array}$ & $\begin{array}{c}8.84^{B} \pm \\
0.10\end{array}$ \\
\hline 48 & $\begin{array}{l}20.22 \\
\pm 0.34\end{array}$ & $\begin{array}{l}16.34 \\
\pm 0.61\end{array}$ & $\begin{array}{c}3.72 \\
\pm 0.13\end{array}$ & $\begin{array}{c}9.45 \\
\pm 0.12\end{array}$ & $\begin{array}{r}12.45^{\mathrm{A}} \\
\pm 0.13\end{array}$ & $\begin{array}{l}29.92 \\
\pm 0.65\end{array}$ & $\begin{array}{l}21.19 \\
\pm 0.72\end{array}$ & $\begin{array}{l}11.85 \\
\pm 0.19\end{array}$ & $\begin{array}{l}15.65 \\
\pm 0.13\end{array}$ & $\begin{array}{c}19.90^{\mathrm{A}} \\
\pm 0.23\end{array}$ & $\begin{array}{l}22.41 \\
\pm 0.21\end{array}$ & $\begin{array}{l}15.33 \\
\pm 0.14\end{array}$ & $\begin{array}{c}5.86 \\
\pm 0.13\end{array}$ & $\begin{array}{l}12.36 \\
\pm 0.10\end{array}$ & $\begin{array}{c}13.99^{A} \\
\pm 0.11\end{array}$ \\
\hline Means & $\begin{array}{l}15.01^{\mathrm{a}} \\
\pm 0.87\end{array}$ & $\begin{array}{l}11.94^{b} \\
\pm 0.46\end{array}$ & $\begin{array}{l}2.43^{d} \\
\pm 0.14\end{array}$ & $\begin{array}{l}6.67^{\mathrm{c}} \\
\pm 0.11\end{array}$ & $8.99^{b}$ & $\begin{array}{l}20.63^{a} \\
\pm 0.23\end{array}$ & $\begin{array}{c}15.29^{b} \\
\pm 0.18\end{array}$ & $\begin{array}{l}7.41^{d} \\
\pm 0.81\end{array}$ & $\begin{array}{l}11.44^{\mathrm{c}} \\
\pm 0.16\end{array}$ & $13.69^{\mathrm{a}}$ & $\begin{array}{l}15.40^{a} \\
\pm 0.12\end{array}$ & $\begin{array}{c}10.29^{b} \\
\pm 0.19\end{array}$ & $\begin{array}{l}3.86^{d} \\
\pm 0.13\end{array}$ & $\begin{array}{c}7.26^{c} \\
\pm 0.17\end{array}$ & $9.20^{b}$ \\
\hline
\end{tabular}

A-C: Means with the different superscripts in the same column, differ significantly $(\mathrm{P}<0.05)$.

a-d: Means with the different superscripts in the same row, differ significantly $(\mathrm{P}<0.05)$.

The highest $(\mathrm{P}<0.05)$ percentage of acrosome damage of spermatozoa was recorded in the control samples and the lowest $(\mathrm{P}<0.05)$ percentage was recorded in the extended semen with $0.4 \mathrm{mM} / 100 \mathrm{ml} \mathrm{GSH}$ in the different extenders. The prolongation of storage time at $5^{\circ} \mathrm{C}$ for up to 48 hours increased significantly $(\mathrm{P}<0.05)$ the percentage of acrosome damage of
Data presented in Table 5 shows that, the percentage of acrosome damage of spermatozoa significantly $(\mathrm{P}<0.05)$ lower in tris-yolk-glucose extender and Non-fat dried milk solids glucose extender than Milkglucose extender.

Supplementation of the extended stallion spermatozoa with GSH up to $0.8 \mathrm{mM} / 100 \mathrm{ml}$ caused decreased significantly $(\mathrm{P}<0.05)$ the percentage of acrosome damage of spermatozoa, during storage at $5^{\circ} \mathrm{C}$ for up to 48 hours in the different extenders. 


\section{DISCUSSION}

Cooled equine semen is used to allow its shipment for variable periods of time. During transportation, the semen should keep the fertility and that depends on storage temperature, composition of extender and spermatic concentration per insemination dose (Heckenbichler et al., 2011). The cooled shipped system that we chose maintains the semen at $16{ }^{\circ} \mathrm{C}$ for $24 \mathrm{~h}$. it is low cost procedure, widely used during the breeding season and allows the shipment of semen for long distances (Melo et al., 2007).

Data present in Table 2 reveled that the percentage of motile stallion spermatozoa in tris-yolk-glucose was high because Tris in addition to its buffering capacity can readily diffuse into the sperm cell and serve as an intracellular buffer. Similar results fount by (Bartlett and Van Demark, 1962). These results may be due to the combination of all beneficial effects of the tris components. The addition of egg yolk which contains phospholipids and lecithin to extenders may be protect the sperm membrane against cold shock (Paulenz et al., 2002). Similarly, Waheed (2001) and Bozkurt et al. (2007) showed that tris-egg yolk extender at $5^{\circ} \mathrm{C}$ was maintaining the motility of stallion spermatozoa. Moreover, Bergeron and Manjunath (2006), Bergeron et al. (2007), Lusignan et al. (2011) and El-Nady (2017) found that, the Nonfat Dried milk extender was good protection to spermatozoa because the milk contains of caseins micelles (the major proteins of milk). In stallion spermatozoa, Ball and Vo (2001) found that the addition of glutathione to semen extender prevented the loss of sperm motility by inhibition of lipid peroxidation caused by reactive oxygen species (ROS). Moreover de Oliveira et al. (2013), Oliveira (2014) and El-Nady (2017) reported that the addition of $2.5 \mathrm{mM}$ glutathione to the freezing extender increased the total motility and the sperm viability of stallion spermatozoa. Similar trend was reported by Bustamante-Filho (2006) in stallion spermatozoa. These results may be accompanied with a precipitous fall in the rate of fructolysis (Mann and LutwakMann, 1981), or may be due to change in spermatozoal morphology and coiled tail thus reducing the spermatozoal flagellum movement (ElHarairy et al., 2011). De Oliveira et al. (2015) found that the advancement of storage time at $16^{\circ} \mathrm{C}$ for up to 36 hours increased significantly $(\mathrm{P}<0.05)$ the percentage of total motility of stallion spermatozoa with different levels of GSH. Moreover, cryopreservation of spermatozoa reduced motility and affects DNA integrity in humen spermatozoa (Branco et al., 2010) and in the Arabian stallion spermatozoa (El-Nady, 2017).

In Table 3 the low percentage of abnormal stallion spermatozoa in Tris-yolk-glucose extender may be due to the beneficial effects of tris extender allowing more protection of spermatozoa, consequently, lowering the dead spermatozoa during storage at $5^{\circ} \mathrm{C}$. El-Nady, (2017) in the Arabian stallion spermatozoa, found that, the lowest $(\mathrm{P}<0.05)$ of dead spermatozoa was recorded with glucose-yolk fructose extender, while the highest $(\mathrm{P}<0.05)$ value was recorded with Milk-glucose extender. The differences in sperm motility between GSH-treated and control samples become more evident upon increasing the storage time. De Oliveira et al. (2013) found that the addition of $2.5 \mathrm{mM}$ glutathione to the freezing extender decreased percentage of dead stallion spermatozoa. However, Zhandi and Ghadimi (2014) and El-Nady (2017) found significantly effects of glutathione on the viability stallion spermatozoa, during storage at $5^{\circ} \mathrm{C}$. El-Nady (2017) indicated that the advancement of storage time at $5^{\circ} \mathrm{C}$ for up to $48 \mathrm{~h}$ increased significantly $(\mathrm{P}<0.05)$ the percentage of dead cooled stallion spermatozoa added with all different concentrations of GSH. These findings may be attributed to the accumulation of lactic acid which exerts a toxic effect on sperm cell and leakage of intracellular enzymes due to the increased membrane permeability (Zeidan, 1994).

Data presented in Table 4 showed that the obtained results for abnormal spermatozoa are in agreement with those of Waheed (2001) who found that, glycineegg yolk extender and Tris extender gave a high viability of stallion's semen than other extenders containing milk. In addition, El-Nady (2017) found that the lowest $(\mathrm{P}<0.05)$ value of the percentage of abnormal spermatozoa was recorded with tris-yolk extender, while the highest value was recorded with milk- glucose extender. Zhandi and Ghadimi (2014), Oliveira et al. (2014) and El-Nady (2017) mentioned that supplementation of INRA82 extender with $5 \mathrm{mM}$ glutathione can preserve stallion spermatozoa during storage at $5^{\circ} \mathrm{C}$ for 48 hours.

The interpretation of the obtained result concerning the abnormal sperm may be due to the increase in sperm metabolic activity, consequently, the increased lactic acid production which in turn exerts a toxic effect on sperm cell (Zeidan, 1994). The advancement of storage time of the stallion extended semen increased the percentage of abnormal spermatozoa (De Oliveira et al., 2013, Zhandi and Ghadimi, 2014 and De Oliveira et al., 2015).

The obtained results of the percentage of acrosome damage are in agreement with those of De Oliveira et al. (2013), El-Nady (2017) and De Oliveira et al. (2015) who found that, the addition of $3.5 \mathrm{mM}$ glutathione to the stallion semen was highly efficient for preservation of viability, acrosome integrity and membrane plasmatic integrity. These results indicated that Glutathione protect the spermatozoa from damage by inhibiting the lipid peroxidation process.

In conclusion, the Arabian stallions spermatozoa extended with Tris-yolk-fructose or Non-fat Dried 
milk solids glucose extender was better than Milkglucose extender, during storage at $5^{\circ} \mathrm{C}$. Addition of glutathione to an extender has more efficiency in maintaining the biological functions and quality of the Arabian stallion spermatozoa under refrigeration condition at $5^{\circ} \mathrm{C}$.

\section{REFERENCES}

Ball, B.A. and VO, A. (2001): Osmotic tolerance of equine spermatozoa and the effects of soluble cryoprotectants on equine sperm motility, viability, and mitochondrial membrane potential. J. Androl., 22(6), 1061-1069.

Bartlett, F. and Van Demark, N. (1962): Effect of diluent composition on survival and fertility of bovine spermatozoa stored in carbonated diluents. J. Dairy Sci., 45(3), 360-367.

Bergeron, A.; Brindle, Y.; Blondin, P. and Manjunath, $P$. (2007): Milk caseins decrease the binding of the major bovine seminal plasma proteins to sperm and prevent lipid loss from the sperm membrane during sperm storage. Biology of Reprod., 77(1), 120-126.

Bergeron, A. and Manjunath, P. (2006): "New insights towards understanding the mechanisms of sperm protection by egg yolk and milk." Molecular Reproduction and Development, 73(10): 1338-1344.

Bozkurt, T.; Türk, G. and Gür, S. (2007): The timedependent motility and longevity of stallion spermatozoa diluted in different spermatozoal oncentrations and extenders during coolstorage. Revue Méd. Vét., 158(02), 67-72.

Branco, C.S.; Garcez, M.E.; Masqualotto, F.F.; Erdtman, B. and Salvador, M. (2010): Resveratorl and ascorbic acid prevent DNA damage induced by cryopreservation in human semen. Cryobiol., 122: 497-506.

Bustamante-Filho, I.C.; Pederzolli, C.D.; Sgaravatti, A.M.; Mattos, R.C.; Dutro-Filho, C.S. and Jobim, M.I.M. (2006): Activity of glutathione peroxidase and catalae in stallion semes during cryopreservation. Animal Reproduction Science, 94: 70-73.

Cocchia, N.; Pasolini, M.P.; Mancini, R.; Petrazzuolo, O.; Cristofaro, I.; Rosapane, I.; Sica, A.; Tortora, G.; Lorizio, R.; Paraggio, G. and Mancini A. (2011): Effect of sod (superoxide dismutase) protein supplementation in semen extenders on motility, viability, acrosome status and ERK (extracelular signal-regulated kinase) protein phosphorylation of chilled stallion spermatozoa. Theriogenology, 75:1201-1210.

Crockett, E.C.; Graham, J.K.; Bruemmer, J.E. and Squires, E.L. (2001): Effect of cooling of equine spermatozoa before freezing on postthaw motility: preliminary results. Theriogenology, 55: 793-803.

De Oliveira, R.A.; de Oliveira, Viu, M.A. and
Gambarini, M.L. (2015): Cooling of equine semen at $16 \mathrm{C}$ for 36 hours with addition of different glutathione concentrations Semina: Ciências Agrárias, Londrina, 36(6), 36993704.

De Oliveira, R.A.; Wolf, C.A.; de Oliveira, Viu, M.A. and Gambarini, M.L. (2013): Addition of glutathione to an extender for frozen equine semen. J. Equine. Vet. Sci., 33(12), 1148-1152.

Duncan, D.B. (1955): Multiple range and multiple F tests. Biometrics, 11(1): 1-42.

El-Harairy, M.A.; Eid, L.N.; Zeidan, A.E.B.; Abd ElSalaam, A.M. and El-Kishk, M. (2011): Quality and fertility of the frozen-thawed bull semen as affected by the different cryoprotectants and glutathione levels. J. Am. Sci., 7(5), 791-801.

El-Nady, I.A. (2017): Some physiological and biochemical studies on the Arabian horses. MSc. Thesis, Animal department, Faculty of Agriculture, Al-Azhar University, Cairo. Egypt.

Hackett, A. and Macpherson, J. (1965): Some staining procedures for spermatozoa. The Canadian Vet. J., 6(3), 55.

Heckenbichler, S.; Deichscl, K. and Aurich (2011): Quality and fertility of cooled-shipped stallion semen at the time of insemination. Theriogenology, 75: 849-856.

Jasko, D.; Hathaway, J.; Schaltenbrand, V.; Simper, W. and Squires, E. (1992): Effect of seminal plasma and egg yolk on motion characteristics of cooled stallion spermatozoa. Theriogenology, 37(6), 1241-1252.

Luberda, Z. (2005): The role of glutathione in mammalian gametes. Reprod. Biol., 5 (1), 5 17.

Lusignan, M.F.; Bergeron, A.; Lafleur, M. and Manjunath, $P$. (2011): The major proteins of bovine seminal plasma interact with caseins and whey proteins of milk extender. Bio. of Reprod., 85(3), 457-464.

Mann, T. and Lutwak-Mann, C. (1981): Biochemistry of Spermatozoa: Chemical and Functional Correlations in Ejaculated Semen, Andrological Aspect. In: Male Reproductive Function and Semen. Springer, SpringerVerlog. Berlin, Heidelberg, London, New York, USA, p.p. 264-268.

Mckinnon, A.O. and Voss, J.L. (1993): Equine Reproduction. $2^{\text {nd }}$ Edition, Lea \& Fabiger, Philadelphia (USA) and London (UK), p 793

Melo, C.M.; Zahn, F.S.; Mortin, I.; Orlandi, C.; DellAquaj, J.A.; Alvarenya, M.A. and Papa, F.O. (2007): Influence of semen storage and cryoprotectant on post-thaw viability and fertility of stallion spermatozoa. J. Equine Vet. Sci., 27: 171- 175.y

Miller, C.D. (2008): Optimizing the use of frozenthawed equine semen. Theriogenology, 70: 463-468. 
Oliveira, R.; Piersanti, R.; Wolf, C.; Viu, M. and Gambarini, M. (2014): Glutathione for the freezing of cooled equine semen, using different protocols. Anim. Reprod., 11(2), 104109.

Paulenz, H.; Söderquist, L.; Pérez-Pé, R. and Berg, K.A. (2002): Effect of different extenders and storage temperatures on sperm viability of liquid ram semen. Theriogenology, 57(2), 823836.

Salisbury, G.W.; Van Demark, N.U. and Lodge, J.R. (1978): Physiology of Reproduction and Artificial Insemination of Cattle. WH Freeman and Company, San Francisco, USA. 14: $400-$ 418.

SAS (2008): SAS Users, Guide. Statistical Analysis System Institute Inc. Editor, Cary, NC

Solouma, G.M.A. (2013): The influence of adding glutathione on semen characteristics of sohagi rams. Egyptian Journal of Sheep \& Goat Sciences, Vol. 8 (2), P 61- 68, 2013.

Waheed, M.M. (2001): Sexual behavior, semen characteristics and processing in the Arabian horse. Ph. D Thesis, Faculty of Vet. Med., Cairo Univ., Cairo, Egypt.

Watson, $P$. (1975): Use of a Giemsa stain to detect changes in acrosomes of frozen ram spermatozoa. The Vet. Record., 97(1), 12-15.

Zeidan, A.E.B. (1994): New aspects in freezing cattle semen. PhD Thesis, Fac. Agric., Zagazig Univ., Zagazig, Egypt.

Zhandi, M. and Ghadimi, V. (2014): Effect of glutathione-supplemented INRA82 extender on miniature Caspian stallion sperm quality during storage at $5^{\circ} \mathrm{C}$. J. Equine. Vet. Sci., 34(5), 606-610.

\section{تاثير اضافة الجلوتاثيون على مخفف السائل المنوى المبرد للخيول العربية

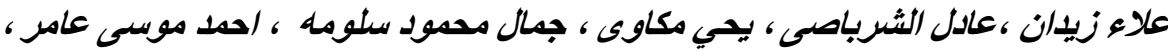

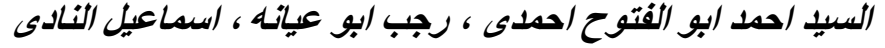

E-mail: gsolouma2@hotmail.com Assiut University web-site: www.aun.edu.eg

تم في هذه الدرراسة جمع ^/ قذفة سائل منوى من ثلاثة خيول عربية تبلغ اعمار ها ه سنوات خلال فترة النشاط الجنسى (الصيف)

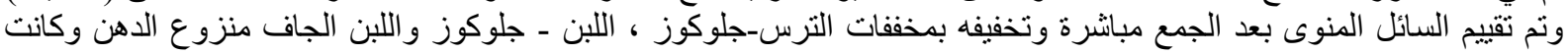

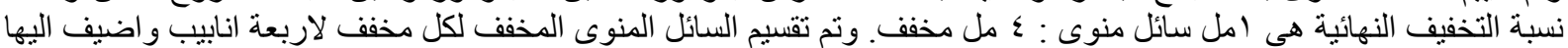

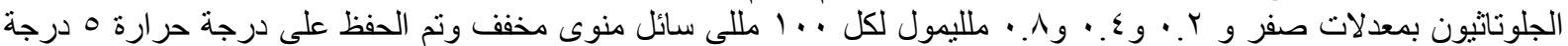

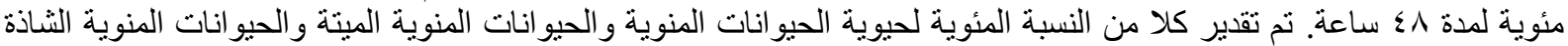
و الحيو انات المنوية تالفة الاكروسوم. تلفير كلاع من



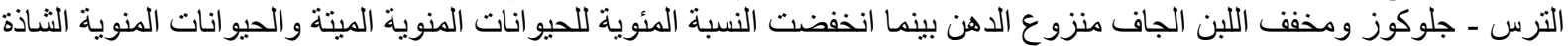

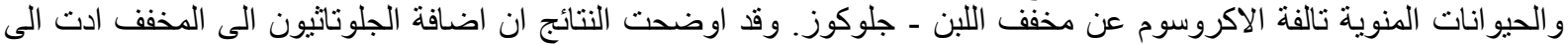

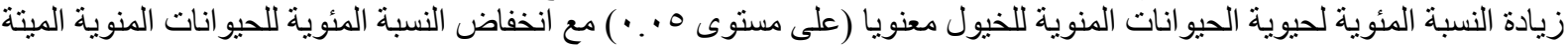

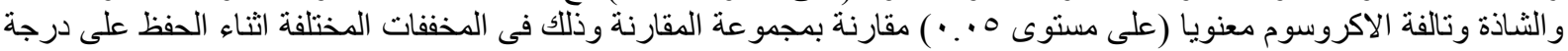





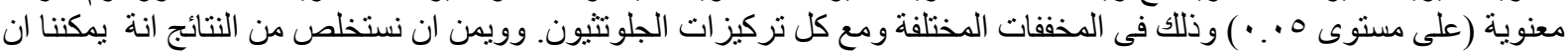

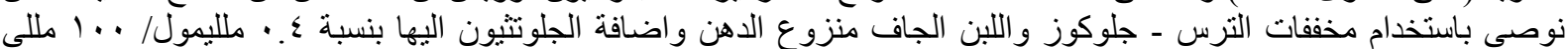



